\title{
INFLUENCE OF UNIAXIAL AND HYDROSTATIC PRESSURES AND A LONGITUDINAL ELECTRIC FIELD ON THE THERMODYNAMIC CHARACTERISTICS OF THE QUASI-ONE-DIMENSIONAL FERROELECTRIC $\mathrm{CsH}_{2} \mathrm{PO}_{4}$
}

\author{
A. S. Vdovych ${ }^{1}$ (D), R. R. Levitskii ${ }^{1}$, I. R. Zachek ${ }^{2}$, A. P. Moina ${ }^{1}$ (D) \\ ${ }^{1}$ Institute for Condensed Matter Physics of the National Academy of Sciences of Ukraine, \\ 1, Svientsitskii St., Lviv, UA-79011, Ukraine, \\ ${ }^{2}$ Lviv Polytechnic National University, \\ 12, Bandery St., Lviv, UA-79013, Ukraine
}

(Received 04 January 2021; in final form 22 April 2021; accepted 11 May 2021; published online 14 July 2021)

\begin{abstract}
We propose a two-sublattice proton ordering model for the quasi-one-dimensional $\mathrm{CsH}_{2} \mathrm{PO}_{4}$ ferroelectric with hydrogen bonds, which takes into account contributions to the energy of the proton subsystem that are linear in the lattice strains $\varepsilon_{1}, \varepsilon_{2}, \varepsilon_{3}$, and $\varepsilon_{5}$. The model also takes into account the dependence of the effective dipole moments of pseudospins on the order parameters, which enables us to coordinate the values of the effective dipole moments in the paraelectric and ferroelectric phases. Within this model in the two-particle cluster approximation for the short-range interactions and in the mean field approximation for the long-range interactions, the behavior of spontaneous polarization, longitudinal dielectric permittivity, piezoelectric and elastic characteristics, and molar heat capacity under the influence of uniaxial and hydrostatic pressure and a longitudinal electric field are investigated.
\end{abstract}

Key words: ferroelectrics, dielectric permittivity, piezoelectric coefficients, pressure effect, electric field effect.

DOI: https://doi.org/10.30970/jps.25.3702

\section{INTRODUCTION}

Investigations of the external pressure and field effects in ferroelectrics are an important problem of ferroelectrics physics. High pressure studies give a possibility to obtain additional valuable information on the peculiarities of the behavior of the physical properties of ferroelectric compounds, as well as to search for new physical effects, which are not observed under zero pressure and zero external field. It should be also underlined that these studies give an opportunity to understand better the mechanisms of phase transitions in these materials.

The ferroelectric with hydrogen bonds $\mathrm{CsH}_{2} \mathrm{PO}_{4}$ (CDP) is an example of a crystal, where the pressure and field effects are essential. In this crystal, there are two structurally non-equivalent types of hydrogen bonds of different lengths (Fig. 1,b). The longer bonds have one equilibrium position for protons, whereas the shorter bonds have two equilibrium positions. They link $\mathrm{PO}_{4}$ groups into chains along the $b$-axis (Fig. 1,a); therefore the crystal is quasi-one-dimensional.

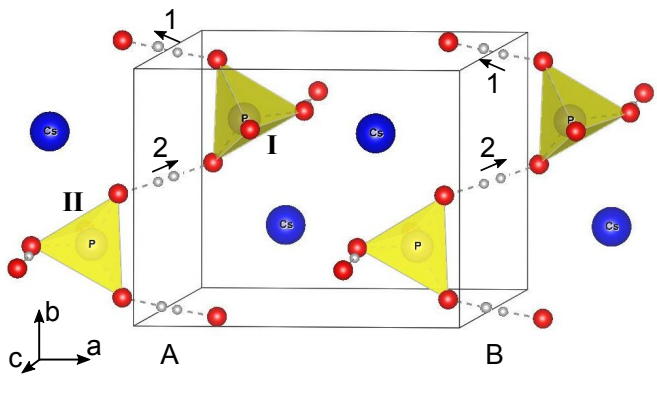

a

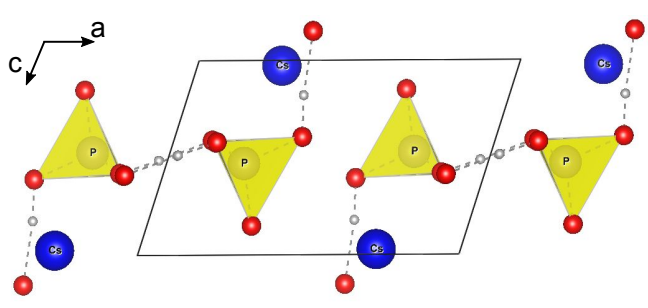

$\mathrm{b}$

Fig. 1. The primitive cell of the $\mathrm{CsH}_{2} \mathrm{PO}_{4}$ crystal in the ferroelectric phase 
At room temperature and zero pressure, the crystal is in the paraelectric phase and has a monoclinic symmetry (the space group $P 2_{1} / m$ ) $[1,2]$. Protons on the shorter bonds occupy two equilibrium positions with equal probability. Below $T_{c}=153 \mathrm{~K}$, the crystal goes into the ferroelectric phase (the space group $P 2_{1}$ ) [3, 4] with spontaneous polarization along the crystallographic $b$-axis, and the protons occupy mainly upper equilibrium positions (Fig. 1, a).

On the basis of dielectric measurements $[5,6]$ it was determined that under pressure $p_{c}=0.33 \mathrm{GPa}$ and $T_{c}^{\mathrm{cr}}=124.6 \mathrm{~K}$ there appear double hysteresis loops, that is, the crystal goes to the antiferroelectric phase. By neutron scattering experiments [7] it has been established that in the antiferroelectric phase the primitive cell of CDP crystal doubles along the $a$-axis, because there appear two sublattices in the form of $b c$-planes, which are polarized antiparallelly along the $b$-axis and alternate along the $a$-axis. The symmetry of the crystal remains monoclinic (the space group $P 2_{1}$ ), and the constants of the double lattice are as follows: $a=15.625 \AA$, $b=6.254 \AA, c=4.886 \AA, \beta=108.08^{\circ}$. A significant shift of $\mathrm{Cs}$ atoms and $\mathrm{PO}_{4}$ groups in $(a, c)$-plane and rotation of the $\mathrm{PO}_{4}$ groups by $36.8^{\circ}$ around the $b$ axis, which goes through the $\mathrm{P}$ atom, occur. The protons on the hydrogen bonds of neighboring sublattices are ordered antiparallelly. At very high pressures, the antiferroelectric phase of a second type (AF2) appears, where two sublattices have a form of chains along the $b$-axis, which are polarized antiparallelly along $b$-axis and have a checkerboard arrangement. The AF2 phase was predicted in [8] on the basis of NMR data [8] and confirmed in [9] on the basis of dielectric constant measurements at low temperature and X-ray diffraction experiments.

Influence of hydrostatic pressure on the transition temperature in the $\mathrm{Cs}\left(\mathrm{H}_{1-x} \mathrm{D}_{x}\right)_{2} \mathrm{PO}_{4}$ ferroelectrics was explored in [5, 6, 9-12].

Results of experimental measurements of the temperature dependence of spontaneous polarization of the $\mathrm{CsH}_{2} \mathrm{PO}_{4}$ crystal at different values of hydrostatic pressure are presented in [6], and of the static longitudinal dielectric permittivity in $[6,9,11,12]$.

The lattice dynamics of $\mathrm{CsH}_{2} \mathrm{PO}_{4}$ and $\mathrm{CsD}_{2} \mathrm{PO}_{4}$ crystals was explored in $[13,14]$. The phonon spectra of these crystals and specific heat were calculated. The obtained temperature variation of the specific heat qualitatively agrees with the experimental data, except for the vicinity of the phase transition. Later, the ab initio calculations have shown an important role of proton tunneling [15], and also the piezoelectric coefficients, elastic constants, and specific heat of $\mathrm{CsH}_{2} \mathrm{PO}_{4}[16,17]$.

An attempt to theoretically describe the paraelectricferroelectric and paraelectric-antiferroelectric phase transition in $\mathrm{CsH}_{2} \mathrm{PO}_{4}$ and $\mathrm{CsD}_{2} \mathrm{PO}_{4}$ as well as experimental data for the dielectric permittivity was done in [18], where the crystal was described as pseudospin Ising chains. However, the description of the experimental data for the dielectric constant by the proposed theory was not considered.

Later [19], using the pseudospin model [18] and the two-particle cluster approximation for the shortrange configuration interactions, the thermodynamic and dynamic characteristics of CDP type ferroelectrics were calculated at different values of hydrostatic pressure. A good agreement of the theory with the experimental data for the dielectric constant and for the pressure dependence of the para-ferroelectric and para-antiferroelectric phase transition temperatures was obtained. However, in the model [18, 19] one cannot calculate the piezoelectric and elastic characteristics of the crystal, and the critical pressure does not depend on temperature.

In [20], the temperature dependences of the lattice strains $\varepsilon_{1}, \varepsilon_{2}, \varepsilon_{3}, \varepsilon_{5}$ were measured. There was also proposed a quasi-one-dimensional Ising model for the $\mathrm{CsH}_{2} \mathrm{PO}_{4}$ crystal, where the interaction parameters were linear functions of these strains. On the basis of this model, the temperature dependences of $\varepsilon_{j}(T)$ were explained. However, this model does not consider the crystal as two sublattices and does not allow one to describe the ferroelectric-antiferroelectric phase transition under high pressures.

In [21], a two-sublattice model of a deformed $\mathrm{CsH}_{2} \mathrm{PO}_{4}$ crystal has been proposed, where the interactions between neighboring pseudospins within a chain are taken into account in the two-particle cluster approximation, whereas the long-range interactions (including the interchain ones) are considered in the mean field approximation. The interaction parameters are taken to be linear functions of the strains $\varepsilon_{j}$. As a result, the temperature dependences of spontaneous polarization, dielectric permittivity, piezoelectric coefficients, and elastic constants were calculated.

In [22], using the model of the deformed $\mathrm{CsH}_{2} \mathrm{PO}_{4}$ crystal, proposed in [21] the effects of hydrostatic pressure on the phase transition temperature, longitudinal static dielectric characteristics of $\mathrm{Cs}\left(\mathrm{H}_{1-x} \mathrm{D}_{x}\right)_{2} \mathrm{PO}_{4}$ crystals were studied.

As is well known, in the presence of the longitudinal field $E_{y}$, the second order phase transition is smeared, and the temperature dependence of the longitudinal permittivity $\varepsilon_{y y}(T)$ has a rounded maximum. At the same time, in $[21,22]$, to calculate the longitudinal dielectric permittivity $\varepsilon_{y y}$, different values for the effective dipole moments in the paraelectric and ferroelectric phases were used. This gives rise to a jump in the $\varepsilon_{y y}(T)$ curve, instead of the rounded maximum expected in presence of the external field $E_{y}$. Therefore, in order to describe the smearing of phase transition, in [23] we have modified the model [21] by assuming that the effective dipole moment on a hydrogen bond depends on the order parameter on this bond, because the order parameter depends on temperature continuously near the phase transition point. The behavior of spontaneous polarization, longitudinal dielectric permittivity, and molar specific heat in the presence of hydrostatic pressure and a longitudinal electric field was explored [23].

In the present paper, we study the behavior of the spontaneous polarization, longitudinal dielectric permittivity, piezoelectric and elastic characteristics, and 
molar specific heat of $\mathrm{CsH}_{2} \mathrm{PO}_{4}$ in the presence of uniaxial and hydrostatic pressure, a longitudinal electric field, considering also the case when the pressures and the electric field are applied simultaneously.

\section{THE MODEL OF THE $\mathrm{CsH}_{2} \mathrm{PO}_{4}$ CRYSTAL}

We study the system of protons in the $\mathrm{CsH}_{2} \mathrm{PO}_{4}$ crystal, moving on the short $\mathrm{O}-\mathrm{H}$...O bonds between the $\mathrm{HPO}_{4}$ groups, which form zigzag chains along the crystallographic $b$-axis of the crystal (see Fig.1).

The cell contains one chain, marked as "A" in Fig. 1. In order to describe the transition to the antiferroelectric phase, under high pressure we shall consider an extended primitive cell, which includes two chains ("A" and "B"). All the "A" chains form the sublattice "A", whereas all the "B" chains form the sublattice "B". Every chain in the primitive cell includes two neighboring $\mathrm{PO}_{4}$ tetrahedra (of type "I" and "IT') together with two short hydrogen bonds (respectively, " 1 " and "2").

Let us ascribe the dipole moments $\mathbf{d}_{q 1}^{\mathrm{A}}, \mathbf{d}_{q 2}^{\mathrm{A}}, \mathbf{d}_{q 1}^{\mathrm{B}}, \mathbf{d}_{q 2}^{\mathrm{B}}$ to the protons on the bonds. The pseudospin variables $\frac{\sigma_{q 1}^{\mathrm{A}}}{2}, \frac{\sigma_{q 2}^{\mathrm{A}}}{2}, \frac{\sigma_{q 1}^{\mathrm{B}}}{2}, \frac{\sigma_{q 2}^{\mathrm{B}}}{2}$ describe the changes associated with reordering of the respective dipole moments of the structure units: $\mathbf{d}_{q 1,2}^{\mathrm{A}, \mathrm{B}}=\boldsymbol{\mu} \frac{\sigma_{q 1,2}^{\mathrm{A}, \mathrm{B}}}{2}$. The mean values $\left\langle\frac{\sigma}{2}\right\rangle=$ $\frac{1}{2}\left(n_{a}-n_{b}\right)$ are related to the differences in occupancies of the two possible positions or the protons on the bonds, $n_{a}$ and $n_{b}$.

The Hamiltonian of the proton subsystem of $\mathrm{CsH}_{2} \mathrm{PO}_{4}$ takes into account the short-range and longrange interactions. Under the stresses maintaining the symmetry of crystal $\sigma_{1}=\sigma_{x x}, \sigma_{2}=\sigma_{y y}, \sigma_{3}=\sigma_{z z}$, $\sigma_{5}=\sigma_{x z}(X \perp(b, c), Y\|b, Z\| c)$, and in the presence of electric field $E_{2}=E_{y}$, it can be written as [23]:

$$
\begin{aligned}
\hat{H} & =N v\left\{\frac{1}{2} \sum_{j, j^{\prime}} c_{j j^{\prime}}^{E 0} \varepsilon_{j} \varepsilon_{j}^{\prime}-\sum_{j} e_{2 j}^{0} E_{2} \varepsilon_{j}-\frac{1}{2} \chi_{22}^{\varepsilon 0} E_{2}^{2}\right\} \\
& -2 w \sum_{q q^{\prime}}\left(\frac{\sigma_{q 1}^{\mathrm{A}}}{2} \frac{\sigma_{q^{\prime} 2}^{\mathrm{A}}}{2}+\frac{\sigma_{q 1}^{\mathrm{B}}}{2} \frac{\sigma_{q^{\prime} 2}^{\mathrm{B}}}{2}\right)\left(\delta_{\mathbf{R}_{q} \mathbf{R}_{q^{\prime}}}+\delta_{\mathbf{R}_{q}+\mathbf{R}_{b}, \mathbf{R}_{q^{\prime}}}\right) \\
& +\hat{H}_{\text {long }}-\sum_{q} \mu_{y} E_{2}\left(\frac{\sigma_{q 1}^{\mathrm{A}}}{2}+\frac{\sigma_{q 2}^{\mathrm{A}}}{2}+\frac{\sigma_{q 1}^{\mathrm{B}}}{2}+\frac{\sigma_{q^{2}}^{\mathrm{B}}}{2}\right) \\
& -3\left\{\sum_{q}\left(\frac{\eta_{1}^{2} \sigma_{q 1}^{\mathrm{A}}}{2}+\frac{\eta_{1}^{2} \sigma_{q 2}^{\mathrm{A}}}{2}+\frac{\eta_{2}^{2} \sigma_{q 1}^{\mathrm{B}}}{2}+\frac{\eta_{2}^{2} \sigma_{q 2}^{\mathrm{B}}}{2}\right)+2 N\left(\eta_{1}^{3}+\eta_{2}^{3}\right)\right\} \mu^{\prime} E_{2},
\end{aligned}
$$

where $N$ is the total number of extended primitive cells of the Bravais lattice; $v$ is their volume.

The first term in (2.1) is the "seed" energy, which corresponds to the heavy ion sublattice and does not explicitly depend on the configuration of the proton subsystem. It includes the elastic, piezolectric, and dielectric parts, expressed in terms of the electric field $E_{2}$, and strains preserving the symmetry of the crystal $\varepsilon_{1}=\varepsilon_{x x}, \varepsilon_{2}=\varepsilon_{y y}, \varepsilon_{3}=\varepsilon_{z z}, \varepsilon_{5}=2 \varepsilon_{x z} . c_{j j^{\prime}}^{E 0}, e_{2 j}^{0}, \chi_{22}^{\varepsilon_{0}^{0}}$ $(j=1,2,3,5)$ are the "seed" elastic constants, coefficients of piezoelectric stress, and dielectric susceptibility of a mechanically clamped crystal.

The second term in (2.1) is the Hamiltonian of the short-range interactions. In $(2.1), \sigma_{q 1,2}^{\mathrm{A}, \mathrm{B}}$ are the $z$ components of the pseudospin operator that describe the state of the bond " 1 " or " 2 " of the chain " $\mathrm{A}$ " or " $\mathrm{B}$ ", in the $q$-th cell, $\mathbf{R}_{b}$ is the lattice vector along the $O Y$-axis. The first and the second Kronecker delta-symbols correspond to the interaction between protons in the chains near the tetrahedra $\mathrm{PO}_{4}$ of type "I" and of type "II", respectively. The contributions to the energy of interactions between pseudospins near tetrahedra of different types are identical.

The parameter $w$, which describes the short-range interactions within the chains, is expanded linearly into a series over the strains $\varepsilon_{j}$

$$
w=w^{0}+\sum_{j} \delta_{2 j} \varepsilon_{j}, \quad j=1,2,3,5 .
$$

The term $\hat{H}_{\text {long }}$ in (2.1) describes the long-range dipole-dipole interactions and indirect (via the lattice vibrations) interactions between protons, taken into account in the mean field approximation

$$
\hat{H}_{\text {long }}=N H^{0}+\hat{H}_{2},
$$

where 


$$
\begin{aligned}
H^{0} & =\frac{1}{8}\left[J_{11}\left(\left\langle\sigma_{1}^{\mathrm{A}}\right\rangle^{2}+\left\langle\sigma_{1}^{\mathrm{B}}\right\rangle^{2}\right)+J_{22}\left(\left\langle\sigma_{2}^{\mathrm{A}}\right\rangle^{2}+\left\langle\sigma_{2}^{\mathrm{B}}\right\rangle^{2}\right)+2 J_{12}\left(\left\langle\sigma_{1}^{\mathrm{A}}\right\rangle\left\langle\sigma_{2}^{\mathrm{A}}\right\rangle+\left\langle\sigma_{1}^{\mathrm{B}}\right\rangle\left\langle\sigma_{2}^{\mathrm{B}}\right\rangle\right)\right] \\
& +\frac{1}{4}\left[K_{11}\left(\sigma_{1}^{\mathrm{A}}\right\rangle\left\langle\sigma_{1}^{\mathrm{B}}\right)+K_{22}\left\langle\sigma_{2}^{\mathrm{A}}\right\rangle\left\langle\sigma_{2}^{\mathrm{B}}\right\rangle+K_{12}\left(\left\langle\sigma_{1}^{\mathrm{A}}\right\rangle\left\langle\sigma_{2}^{\mathrm{B}}\right\rangle+\left\langle\sigma_{1}^{\mathrm{B}}\right\rangle\left\langle\sigma_{2}^{\mathrm{A}}\right\rangle\right)\right], \\
\hat{H}_{2} & =-\sum_{q}\left\{\left(J_{11}\left\langle\sigma_{1}^{\mathrm{A}}\right\rangle+J_{12}\left\langle\sigma_{2}^{\mathrm{A}}\right\rangle+K_{11}\left\langle\sigma_{1}^{\mathrm{B}}\right\rangle+K_{12}\left\langle\sigma_{2}^{\mathrm{B}}\right\rangle\right) \frac{\sigma_{q 1}^{\mathrm{A}}}{4}+\left(J_{12}\left\langle\sigma_{1}^{\mathrm{A}}\right\rangle+J_{22}\left\langle\sigma_{2}^{\mathrm{A}}\right\rangle+K_{12}\left\langle\sigma_{1}^{\mathrm{B}}\right\rangle+K_{22}\left\langle\sigma_{2}^{\mathrm{B}}\right\rangle\right) \frac{\sigma_{q 2}^{\mathrm{A}}}{4}\right. \\
& \left.+\left(J_{11}\left\langle\sigma_{1}^{\mathrm{B}}\right\rangle+J_{12}\left\langle\sigma_{2}^{\mathrm{B}}\right\rangle+K_{11}\left\langle\sigma_{1}^{\mathrm{A}}\right\rangle+K_{12}\left\langle\sigma_{2}^{\mathrm{A}}\right\rangle\right) \frac{\sigma_{q 1}^{\mathrm{B}}}{4}+\left(J_{12}\left\langle\sigma_{1}^{\mathrm{B}}\right\rangle+J_{22}\left\langle\sigma_{2}^{\mathrm{B}}\right\rangle+K_{12}\left\langle\sigma_{1}^{\mathrm{A}}\right\rangle+K_{22}\left\langle\sigma_{2}^{\mathrm{A}}\right\rangle\right) \frac{\sigma_{q 2}^{\mathrm{B}}}{4}\right\} .
\end{aligned}
$$

Here the parameters $J_{f f^{\prime}}=\sum_{\mathbf{R}_{q}-\mathbf{R}_{q^{\prime}}} J_{f f^{\prime}}\left(q q^{\prime}\right)$ and $K_{f f^{\prime}}=$ $\sum_{\mathbf{R}_{q}-\mathbf{R}_{q^{\prime}}} K_{f f^{\prime}}\left(q q^{\prime}\right)$ are the Fourier transforms of the longrange interaction constants at $\mathbf{k}=0$.

Let us expand the parameters $J_{f f^{\prime}}$ i $K_{f f^{\prime}}$ in series over the strains $\varepsilon_{j}$ up to the linear terms

$$
\begin{aligned}
& J_{11}=J_{22}=J_{1}+\sum_{j} \bar{\varphi}_{1 j} \varepsilon_{j}, \\
& J_{12}=J_{21}=J_{2}+\sum_{j} \bar{\varphi}_{2 j} \varepsilon_{j}, \\
& K_{11}=K_{22}=K_{1}+\sum_{j} \varphi_{1 j} \varepsilon_{j}, \\
& K_{12}=K_{21}=K_{2}+\sum_{j} \varphi_{2 j} \varepsilon_{j} .
\end{aligned}
$$

Taking into account the following symmetry of pseudospins in the chains

$$
\left\langle\sigma_{q 1}^{\mathrm{A}}\right\rangle=\left\langle\sigma_{q 2}^{\mathrm{A}}\right\rangle=\eta_{1}, \quad\left\langle\sigma_{q 1}^{\mathrm{B}}\right\rangle=\left\langle\sigma_{q 2}^{\mathrm{B}}\right\rangle=\eta_{2}
$$

we obtain the expressions for (2.4), (2.5) as

$$
\hat{H}^{0}=\nu_{1}\left(\eta_{1}^{2}+\eta_{2}^{2}\right)+2 \nu_{2} \eta_{1} \eta_{2}
$$

$$
\begin{aligned}
\hat{H}_{2} & =\sum_{q}\left\{-\left(2 \nu_{1} \eta_{1}+2 \nu_{2} \eta_{2}\right)\left(\frac{\sigma_{q 1}^{\mathrm{A}}}{2}+\frac{\sigma_{q 2}^{\mathrm{A}}}{2}\right)\right. \\
& \left.-\left(2 \nu_{2} \eta_{1}+2 \nu_{1} \eta_{2}\right)\left(\frac{\sigma_{q 1}^{\mathrm{B}}}{2}+\frac{\sigma_{q 2}^{\mathrm{B}}}{2}\right)\right\} .
\end{aligned}
$$

Here we used the following notations

$$
\begin{aligned}
\nu_{1} & =\frac{1}{8}\left(J_{11}+J_{22}+2 J_{12}\right)=\nu_{1}^{0}+\sum_{j} \psi_{j 1} \varepsilon_{j}, \\
\nu_{1}^{0} & =\frac{1}{4}\left(J_{1}+J_{2}\right), \psi_{j 1}=\frac{1}{4}\left(\bar{\varphi}_{1 j}+\varphi_{1 j}\right) \\
\nu_{2} & =\frac{1}{8}\left(K_{11}+K_{22}+2 K_{12}\right)=\nu_{2}^{0}+\sum_{j} \psi_{j 2} \varepsilon_{j}, \\
\nu_{2}^{0} & =\frac{1}{4}\left(K_{1}+K_{2}\right), \psi_{j 2}=\frac{1}{4}\left(\bar{\varphi}_{2 j}+\varphi_{2 j}\right) .
\end{aligned}
$$

The fourth term in (2.1) describes the interactions of pseudospins with the external electric field, whereas the term $\hat{H}_{E}^{\prime}$ takes into account the abovementioned dependence of effective dipole moments on the mean value of pseudospin $s_{f}$

The two-particle cluster approximation for short-range interactions is used to calculate the thermodynamic characteristics of CDP. In this approximation, the thermodynamic potential is given by

$$
\begin{aligned}
G & =N U_{\text {seed }}+N H^{0}+2 N\left(\eta_{1}^{3}+\eta_{2}^{3}\right) \mu^{\prime} E_{2}-N v \sum_{j} \sigma_{j} \varepsilon_{j} \\
& -k_{\mathrm{B}} T \sum_{q}\left\{2 \ln \mathrm{Sp} e^{-\beta \hat{H}_{q}^{(2)}}-\ln \mathrm{Sp} e^{-\beta \hat{H}_{q}^{(1) A}}-\ln \mathrm{Sp} e^{-\beta \hat{H}_{q}^{(1) B}}\right\},
\end{aligned}
$$

where $\beta=\frac{1}{k_{\mathrm{B}} T}, k_{\mathrm{B}}$ is the Boltzmann constant; $\hat{H}_{q}^{(2)}, \hat{H}_{q}^{(1) A}, \hat{H}_{q}^{(1) B}$ are two-particle and one-particle Hamiltonians 
given by

$$
\begin{aligned}
& \hat{H}_{q}^{(2)}=-2 w\left(\frac{\sigma_{q 1}^{\mathrm{A}}}{2} \frac{\sigma_{q 2}^{\mathrm{A}}}{2}+\frac{\sigma_{q 1}^{\mathrm{B}}}{2} \frac{\sigma_{q 2}^{\mathrm{B}}}{2}\right)-\frac{y_{1}}{\beta}\left(\frac{\sigma_{q 1}^{\mathrm{A}}}{2}+\frac{\sigma_{q 2}^{\mathrm{A}}}{2}\right)-\frac{y_{2}}{\beta}\left(\frac{\sigma_{q 1}^{\mathrm{B}}}{2}+\frac{\sigma_{q 2}^{\mathrm{B}}}{2}\right), \\
& \hat{H}_{q}^{(1) A}=-\frac{\bar{y}_{1}}{\beta}\left(\frac{\sigma_{q 1}^{\mathrm{A}}}{2}+\frac{\sigma_{q 2}^{\mathrm{A}}}{2}\right), \quad \hat{H}_{q}^{(1) B}=-\frac{\bar{y}_{2}}{\beta}\left(\frac{\sigma_{q 1}^{\mathrm{B}}}{2}+\frac{\sigma_{q 2}^{\mathrm{B}}}{2}\right),
\end{aligned}
$$

where the following notations are used

$$
\begin{aligned}
& y_{1}=\beta \Delta_{1}+2 \beta \nu_{1} \eta_{1}+2 \beta \nu_{2} \eta_{2}+\beta\left(\mu_{y} E_{2}+3 \eta_{1}^{2} \mu^{\prime} E_{2}\right), \\
& y_{2}=\beta \Delta_{2}+2 \beta \nu_{2} \eta_{1}+2 \beta \nu_{1} \eta_{2}+\beta\left(\mu_{y} E_{2}+3 \eta_{2}^{2} \mu^{\prime} E_{2}\right), \\
& \bar{y}_{1}=\beta \Delta_{1}+y_{1}, \quad \bar{y}_{2}=\beta \Delta_{2}+y_{2} .
\end{aligned}
$$

Here $\Delta_{l}$ are the effective fields created by the neighboring bonds from outside the cluster. In the cluster approximation, these fields can be determined from the condition of the minimum of thermodynamic potential $\partial G / \partial \Delta_{l}=0$, yielding the self-consistency condition, which states that the mean values of the pseudospins $\left\langle\sigma_{q f}^{\mathrm{A}, \mathrm{B}}\right\rangle$ calculated using two-particle and one-particle Gibbs distributions should coincide

$$
\begin{aligned}
& \eta_{1}=\frac{\operatorname{Sp} \sigma_{q f}^{\mathrm{A}} e^{-\beta \hat{H}_{q}^{(2)}}}{\operatorname{Sp} e^{-\beta \hat{H}_{q}^{(2)}}}=\frac{\operatorname{Sp} \sigma_{q f}^{\mathrm{A}} e^{-\beta \hat{H}_{q}^{(1) \mathrm{A}}}}{\mathrm{Sp} e^{-\beta \hat{H}_{q}^{(1) \mathrm{A}}}}, \\
& \eta_{2}=\frac{\operatorname{Sp} \sigma_{q f}^{\mathrm{B}} e^{-\beta \hat{H}_{q}^{(2)}}}{\operatorname{Sp} e^{-\beta \hat{H}_{q}^{(2)}}}=\frac{\operatorname{Sp} \sigma_{q f}^{\mathrm{B}} e^{-\beta \hat{H}_{q}^{(1) \mathrm{B}}}}{\mathrm{Sp} e^{-\beta \hat{H}_{q}^{(1) \mathrm{B}}}} .
\end{aligned}
$$

Hence, on the basis (2.16) with taking into account of (2.6), (2.12) and (2.13), we obtain the expressions for the order parameters

$$
\begin{aligned}
\eta_{1} & =\frac{1}{D}\left[\sinh \left(y_{1}+y_{2}\right)+\sinh \left(y_{1}-y_{2}\right)+2 a \sinh y_{1}\right] \\
& =\tanh \frac{\bar{y}_{1}}{2}, \\
\eta_{2} & =\frac{1}{D}\left[\sinh \left(y_{1}+y_{2}\right)-\sinh \left(y_{1}-y_{2}\right)+2 a \sinh y_{2}\right] \\
& =\tanh \frac{\bar{y}_{2}}{2},
\end{aligned}
$$

(2.16) where the following notations are used

$$
\begin{aligned}
& D=\cosh \left(y_{1}+y_{2}\right)+\cosh \left(y_{1}-y_{2}\right)+2 a \cosh y_{1}+2 a \cosh y_{2}+2 a^{2}, \\
& a=e^{-\frac{w}{k_{\mathrm{B}} T}} .
\end{aligned}
$$

Excluding the cluster fields $\Delta_{l}$ from the expression $\eta_{l}=\tanh \left(\bar{y}_{l} / 2\right)$ (see (2.17)), we write down (2.14), (2.15) as

$$
\begin{aligned}
& y_{1}=\frac{1}{2} \ln \frac{1+\eta_{1}}{1-\eta_{1}}+\beta \nu_{1} \eta_{1}+\beta \nu_{2} \eta_{2}+\frac{1}{2} \beta\left(\mu_{y} E_{2}+3 \eta_{1}^{2} \mu^{\prime} E_{2}\right), \\
& y_{2}=\frac{1}{2} \ln \frac{1+\eta_{2}}{1-\eta_{2}}+\beta \nu_{2} \eta_{1}+\beta \nu_{1} \eta_{2}+\frac{1}{2} \beta\left(\mu_{y} E_{2}+3 \eta_{2}^{2} \mu^{\prime} E_{2}\right) .
\end{aligned}
$$

\section{THE STATIC LONGITUDINAL DIELECTRIC, PIEZOELECTRIC, ELASTIC, AND THERMAL CHARACTERISTICS OF CDP}

Using (2.11), we write the thermodynamic potential per one extended primitive cell as

$$
\begin{aligned}
g & =U_{\text {seed }}+H^{0}+2\left(\eta_{1}^{3}+\eta_{2}^{3}\right) \mu^{\prime} E_{2}+2 k_{\mathrm{B}} T \ln 2-2 w-v \sum_{j} \sigma_{j} \varepsilon_{j} \\
& -k_{\mathrm{B}} T \ln \left(1-\eta_{1}^{2}\right)-k_{\mathrm{B}} T \ln \left(1-\eta_{2}^{2}\right)-2 k_{\mathrm{B}} T \ln D .
\end{aligned}
$$

Using the equilibrium condition

$$
\left(\frac{\partial g}{\partial \varepsilon_{j}}\right)_{E_{2}}=0
$$


we obtain a set of equations for the strains $\varepsilon_{j}$ :

$$
\sigma_{j}=c_{j 1}^{E 0} \varepsilon_{1}+c_{j 2}^{E 0} \varepsilon_{2}+c_{j 3}^{E 0} \varepsilon_{3}+c_{j 5}^{E 0} \varepsilon_{5}-e_{2 j}^{0} E_{2}-\frac{2 \delta_{j}}{v}+\frac{4 \delta_{j}}{v D} M-\frac{1}{v} \psi_{j 1}\left(\eta_{1}^{2}+\eta_{2}^{2}\right)-\frac{2}{v} \psi_{j 2} \eta_{1} \eta_{2},
$$

where

$$
M=\left[a \cosh y_{1}+a \cosh y_{2}+2 a^{2}\right] .
$$

For hydrostatic pressure, the stresses in (3.2) are $\sigma_{1}=\sigma_{2}=\sigma_{3}=-p_{\mathrm{h}}, \sigma_{5}=0$. For the uniaxial stresses applied along the axis $a, \sigma_{1}=-p_{1}, \sigma_{2}=\sigma_{3}=\sigma_{5}=0$, along the axis $b, \sigma_{2}=-p_{2}, \sigma_{1}=\sigma_{3}=\sigma_{5}=0$, along the axis $c$, $\sigma_{3}=-p_{3}, \sigma_{1}=\sigma_{2}=\sigma_{5}=0$. Hence we obtained the set of equations (2.17), (3.2) for the order parameters $\eta_{f}$ and strains $\varepsilon_{i}, \varepsilon_{5}$.

On the basis of thermodynamic potential (3.1) we easily obtain expressions for different thermodynamic characteristics. In particular, the expression for longitudinal polarization $P_{2}$ reads

$$
P_{2}=-\left(\frac{\partial g}{\partial E_{2}}\right)_{\sigma_{i}}=\sum_{j} e_{2 j}^{0} \varepsilon_{j}+\chi_{22}^{\varepsilon 0} E_{2}+\frac{\mu_{y}}{v}\left(\eta_{1}+\eta_{2}\right)+\frac{\mu^{\prime}}{v}\left(\eta_{1}^{3}+\eta_{2}^{3}\right) .
$$

The isothermal static dielectric susceptibility of a mechanically clamped crystal is

$$
\begin{aligned}
\chi_{22}^{\varepsilon T} & =\left(\frac{\partial P_{2}}{\partial E_{2}}\right)_{\varepsilon_{i}}=\chi_{22}^{\varepsilon 0}+\frac{\beta \tilde{\mu}_{1 y}^{2}}{2 v \Delta}\left\{D\left(\varkappa_{11}+\varkappa_{12}\left(\tilde{\varphi}_{2}-\beta \nu_{2}\right)\left(\varkappa_{11} \varkappa_{22}-\varkappa_{12}^{2}\right)\right\}\right. \\
& +\frac{\beta \tilde{\mu}_{2 y}^{2}}{2 v \Delta}\left\{D\left(\varkappa_{12}+\varkappa_{22}\right)-\left(\tilde{\varphi}_{1}-\beta \nu_{2}\right)\left(\varkappa_{11} \varkappa_{22}-\varkappa_{12}^{2}\right)\right\},
\end{aligned}
$$

where the following notations are used

$$
\begin{aligned}
& \Delta=D^{2}-D\left[\tilde{\varphi}_{1} \varkappa_{11}+\tilde{\varphi}_{2} \varkappa_{22}+2 \beta \nu_{2} \varkappa_{12}\right]+\left[\tilde{\varphi}_{1} \tilde{\varphi}_{2}-\left(\beta \nu_{2}\right)^{2}\right]\left(\varkappa_{11} \varkappa_{22}-\varkappa_{12}^{2}\right) \\
& \tilde{\varphi}_{1}=\varphi_{1}+3 \eta_{1} \beta \mu^{\prime} E_{2}, \quad \tilde{\varphi}_{2}=\varphi_{2}+3 \eta_{2} \beta \mu^{\prime} E_{2} \\
& \varphi_{1}=\frac{1}{1-\eta_{1}^{2}}+\beta \nu_{1}, \varphi_{2}=\frac{1}{1-\eta_{2}^{2}}+\beta \nu_{1} \\
& \tilde{\mu}_{1 y}=\mu_{y}+3 \mu^{\prime} \eta_{1}^{2}, \quad \tilde{\mu}_{2 y}=\mu_{y}+3 \mu^{\prime} \eta_{2}^{2} \\
& \varkappa_{11}=\cosh \left(y_{1}+y_{2}\right)+\cosh \left(y_{1}-y_{2}\right)+2 a \cosh y_{1}-\eta_{1}^{2} D \\
& \varkappa_{12}=\cosh \left(y_{1}+y_{2}\right)-\cosh \left(y_{1}-y_{2}\right)-\eta_{1} \eta_{2} D \\
& \varkappa_{22}=\cosh \left(y_{1}+y_{2}\right)+\cosh \left(y_{1}-y_{2}\right)+2 a \cosh y_{2}-\eta_{2}^{2} D
\end{aligned}
$$

The isothermal coefficients of piezoelectric stress are

$$
e_{2 l}=\left(\frac{\partial P_{2}}{\partial \varepsilon_{l}}\right)_{E_{2}}=e_{2 l}^{0}+\frac{1}{v}\left(\tilde{\mu}_{1 y} \eta_{1}^{\prime(l)}+\tilde{\mu}_{2 y} \eta_{2}^{\prime(l)}\right), \quad l=1,2,3,5,
$$

where the following notations are used

$$
\begin{aligned}
\eta_{1}^{\prime(l)} & =\frac{\beta}{\Delta}\left\{\left(\psi_{l 1} \eta_{1}+\psi_{l 2} \eta_{2}\right)\left[D\left(\varkappa_{11}+\varkappa_{12}\right)-\left(\tilde{\varphi}_{2}-\beta \nu_{2}\right)\left(\varkappa_{11} \varkappa_{22}-\varkappa_{12}^{2}\right)\right]\right. \\
& \left.-\delta_{l}\left[D \rho_{1}-\rho_{1}\left(\beta \nu_{2} \varkappa_{12}+\tilde{\varphi}_{2} \varkappa_{22}\right)+\rho_{2}\left(\beta \nu_{2} \varkappa_{11}+\tilde{\varphi}_{2} \varkappa_{12}\right)\right]\right\}, \\
\eta_{2}^{\prime(l)} & =\frac{\beta}{\Delta}\left\{\left(\psi_{l 2} \eta_{1}+\psi_{l 1} \eta_{2}\right)\left[D\left(\varkappa_{22}+\varkappa_{12}\right)--\left(\tilde{\varphi}_{1}-\beta \nu_{2}\right)\left(\varkappa_{11} \varkappa_{22}-\varkappa_{12}^{2}\right)\right]\right. \\
& \left.-\delta_{l}\left[D \rho_{2}+\rho_{1}\left(\beta \nu_{2} \varkappa_{22}+\tilde{\varphi}_{1} \varkappa_{12}\right)-\rho_{2}\left(\beta \nu_{2} \varkappa_{12}+\tilde{\varphi}_{1} \varkappa_{11}\right)\right]\right\}, \\
\rho_{1} & =2 a \sinh y_{1}-\eta_{1}\left[2 a \cosh y_{1}+2 a \cosh y_{2}+4 a^{2}\right], \\
\rho_{2} & =2 a \sinh y_{2}-\eta_{2}\left[2 a \cosh y_{1}+2 a \cosh y_{2}+4 a^{2}\right] .
\end{aligned}
$$


The constants of piezoelectric stress are obtained by differentiating the electric field over the strains at constant polarization

$$
h_{2 i}=-\left(\frac{\partial E_{2}}{\partial \varepsilon_{i}}\right)_{P_{2}}=\frac{e_{2 i}}{\chi_{22}^{\varepsilon}} .
$$

The calculations of the isothermal elastic constants at a constant field yield

$$
\begin{aligned}
c_{i l}^{E} & =\left(\frac{\partial \sigma_{i}}{\partial \varepsilon_{l}}\right)_{E_{2}}=c_{i l}^{E 0}-\frac{2}{v}\left(\psi_{i 1} \eta_{1}+\psi_{i 2} \eta_{2}+\frac{\delta_{i}}{D} \varkappa_{1}^{c} \tilde{\varphi}_{1}+\frac{\delta_{i}}{D} \varkappa_{2}^{c} \beta \nu_{2}\right) \eta_{1}^{(l)} \\
& -\frac{2}{v}\left(\psi_{i 1} \eta_{2}+\psi_{i 2} \eta_{1}+\frac{\delta_{i}}{D} \varkappa_{1}^{c} \beta \nu_{2}+\frac{\delta_{i}}{D} \varkappa_{2}^{c} \tilde{\varphi}_{2}\right) \eta_{2}^{(l)} \\
& -\frac{2 \beta \delta_{i}}{v_{2} D}\left[\psi_{l 1}\left(\varkappa_{1}^{c} \eta_{1}+\varkappa_{2}^{c} \eta_{2}\right)+\psi_{l 2}\left(\varkappa_{2}^{c} \eta_{1}+\varkappa_{1}^{c} \eta_{1}\right)\right]-\frac{4 \beta \delta_{i} \delta_{l}}{D} \rho^{c}
\end{aligned}
$$

where

$$
\begin{aligned}
& \varkappa_{1}^{c}=\sinh \left(y_{1}+y_{2}\right)+\sinh \left(y_{1}-y_{2}\right)-\eta_{1}\left[\cosh \left(y_{1}+y_{2}\right)+\cosh \left(y_{1}-y_{2}\right)-2 a^{2}\right], \\
& \varkappa_{2}^{c}=\sinh \left(y_{1}+y_{2}\right)-\sinh \left(y_{1}-y_{2}\right)-\eta_{2}\left[\cosh \left(y_{1}+y_{2}\right)+\cosh \left(y_{1}-y_{2}\right)-2 a^{2}\right], \\
& \rho^{c}=2 a^{2}+\frac{\left[\cosh \left(y_{1}+y_{2}\right)+\cosh \left(y_{1}-y_{2}\right)-2 a^{2}\right]}{D}\left[a \cosh y_{1}+a \cosh y_{2}+2 a^{2}\right] .
\end{aligned}
$$

The other dielectric, piezoelectric, and elastic characteristics of $\mathrm{CsH}_{2} \mathrm{PO}_{4}$ can be obtained from those found above. Thus, the isothermal coefficients of the piezoelectric strain are

$$
d_{2 i}=\sum_{j} s_{i j}^{E} e_{2 j}, \quad i, j=1,2,3,5 .
$$

The matrix of the isothermal elastic compliances at a constant field $s_{i j}^{E}$ is inverse to the matrix of the elastic constants $c_{i j}^{E}$

$$
\widehat{C^{E}}=\left(\begin{array}{cccc}
c_{11}^{E} & c_{12}^{E} & c_{13}^{E} & c_{15}^{E} \\
c_{12}^{E} & c_{22}^{E} & c_{23}^{E} & c_{25}^{E} \\
c_{13}^{E} & c_{23}^{E} & c_{33}^{E} & c_{35}^{E} \\
c_{15}^{E} & c_{25}^{E} & c_{35}^{E} & c_{55}^{E}
\end{array}\right), \quad \widehat{S^{E}}=\left(\widehat{C^{E}}\right)^{-1}
$$

The isothermal constants of the piezoelectric strain are

$$
g_{2 i}=\sum_{j} s_{i j}^{E} h_{2 j}
$$

The molar heat capacity of the proton subsystem of CDP at constant pressure can be found by numerically differentiating the thermodynamic potential:

$$
\Delta C_{p}=-\frac{N_{\mathrm{A}} T}{4}\left(\frac{\partial^{2} g}{\partial T^{2}}\right)_{\sigma_{j}} .
$$

where $N_{\mathrm{A}}$ is the Avogadro constant.

\section{COMPARISON OF THE THEORETICAL RESULTS WITH THE EXPERIMENTAL DATA. DISCUSSION}

The theory parameters are determined from the condition of agreement of the calculated characteristics with experimental data for the temperature dependences of spontaneous polarization $P_{2}(T)$ and dielectric permittivity $\varepsilon_{22}(T)$ at different values of hydrostatic pressure [6], spontaneous strains $\varepsilon_{j}$ [20], molar heat capacity [24], and elastic constants [25]; as well as with the results of the ab-initio calculations of the lattice contributions to the molar heat capacity [16] and dielectric permittivity [17].

The parameters of short-range interactions $w_{0}$ and long-range interactions $\nu_{1}^{0}$ ("intra-sublattice"), $\nu_{2}^{0}$ ("intersublattice") mainly determine the phase transition temperature from the paraelectric to the ferroelectric phase in the absence of external pressure and field; the order of the phase transition, and the shape of spontaneous polarization. Their optimum values are $w_{0} / k_{\mathrm{B}}=650 \mathrm{~K}, \nu_{1}^{0} / k_{\mathrm{B}}=1.50 \mathrm{~K}, \nu_{2}^{0} / k_{\mathrm{B}}=0.23 \mathrm{~K}$.

To determine the deformational potentials $\delta_{j}$ [see (2.2)] and $\psi_{j 1}(2.9), \psi_{j 2}(2.10)$, it is necessary to use experimental data for the shift of the phase transition temperature under hydrostatic and uniaxial pressures, as well as the data for the temperature dependences of spontaneous strains $\varepsilon_{j}$, piezoelectric coefficients, and elastic constants. Unfortunately, the only available data are those for the spontaneous strains and hydrostatic pressure effect on the dielectric characteristics. As a result, experimental data for the strains and dielectric characteristics can be described using many different combinations of the parameters $\psi_{j 1}, \psi_{j 2}$. Therefore, for the sake of simplicity, we chose $\psi_{j 2}$ to be proporti- 
onal to $\psi_{j 1}$. The optimum values of the deformational potentials were determined in [23]. Their values are $\delta_{1} / k_{\mathrm{B}}=1214 \mathrm{~K}, \delta_{2} / k_{\mathrm{B}}=454 \mathrm{~K}, \delta_{3} / k_{\mathrm{B}}=1728 \mathrm{~K}$, $\delta_{5} / k_{\mathrm{B}}=1214 \mathrm{~K}, \delta_{5} / k_{\mathrm{B}}=-13 \mathrm{~K} ; \psi_{11} / k_{\mathrm{B}}=92.2 \mathrm{~K}$, $\psi_{21} / k_{\mathrm{B}}=23.2 \mathrm{~K}, \psi_{31} / k_{\mathrm{B}}=139.7 \mathrm{~K}, \psi_{51} / k_{\mathrm{B}}=5.5 \mathrm{~K}$; $\psi_{j 2}=\frac{1}{3} \psi_{j 1}$.

The effective dipole moment in the paraelectric phase is found by fitting the calculated curve $\varepsilon_{22}(T)$ to the experimental data. We consider it to be dependent on the value of hydrostatic pressure $p$, that is, $\mu_{y}=\mu_{y}^{0}\left(1-k_{p} p\right)$, where $\mu_{y}^{0}=2.63 \cdot 10^{-18} \mathrm{esu} \cdot \mathrm{cm}, k_{p}=0.4 \cdot 10^{-10} \mathrm{~cm}^{2} / \mathrm{dyn}$. The correction to effective dipole moment $\mu^{\prime}=-0.43$. $10^{-18} \mathrm{esu} \cdot \mathrm{cm}$ is found by fitting the calculated saturation polarization to the experimental data.

The "seed" dielectric susceptibility $\chi_{22}^{\varepsilon 0}$, coefficients of piezoelectric stress $e_{2 j}^{0}$, and elastic constants $c_{i j}^{E 0}$ are found by fitting to the experimental data at temperatures far from the phase transition temperature $T_{c}$. Their values are as follows $\chi_{22}^{\varepsilon 0}=0.443$ [17]; $e_{2 j}^{0}=0 \frac{\mathrm{esu}}{\mathrm{cm}^{2}}$; $c_{11}^{0 E}=28,83 \cdot 10^{10} \frac{\mathrm{dyn}}{\mathrm{cm}^{2}}, c_{12}^{E 0}=11,4 \cdot 10^{10} \frac{\mathrm{dyn}}{\mathrm{cm}^{2}}, c_{13}^{E 0}=$ $42,87 \cdot 10^{10} \frac{\mathrm{dyn}}{\mathrm{cm}^{2}}, c_{22}^{E 0}=26,67 \cdot 10^{10} \frac{\mathrm{dyn}}{\mathrm{cm}^{2}}, c_{23}^{E 0}=14,5 \cdot 10^{10}$ $\frac{\mathrm{dyn}}{\mathrm{cm}^{2}}, c_{33}^{E 0}=65,45 \cdot 10^{10} \frac{\mathrm{dyn}}{\mathrm{cm}^{2}}, c_{15}^{E 0}=5,13 \cdot 10^{10} \frac{\mathrm{dyn}}{\mathrm{cm}^{2}}, c_{25}^{E 0}=$ $8,4 \cdot 10^{10} \frac{\mathrm{dyn}}{\mathrm{cm}^{2}}, c_{35}^{E 0}=7,50 \cdot 10^{10} \frac{\mathrm{dyn}}{\mathrm{cm}^{2}}, c_{55}^{E 0}=5,20 \cdot 10^{10}$ $\frac{\mathrm{dyn}}{\mathrm{cm}^{2}}$.

The volume of the extended primitive cell is $v=0.467$. $10^{-21} \mathrm{~cm}^{3}[7]$.

Let us consider now the obtained results.

The effects of uniaxial and hydrostatic pressure are determined, mainly, by the pressure-induced behavior of the lattice strains $\varepsilon_{j}$. The temperature dependences of the strains at ambient pressure and in presence of uniaxial pressures are shown in Fig. 2 and in the presence of hydrostatic pressure in Fig. 3.
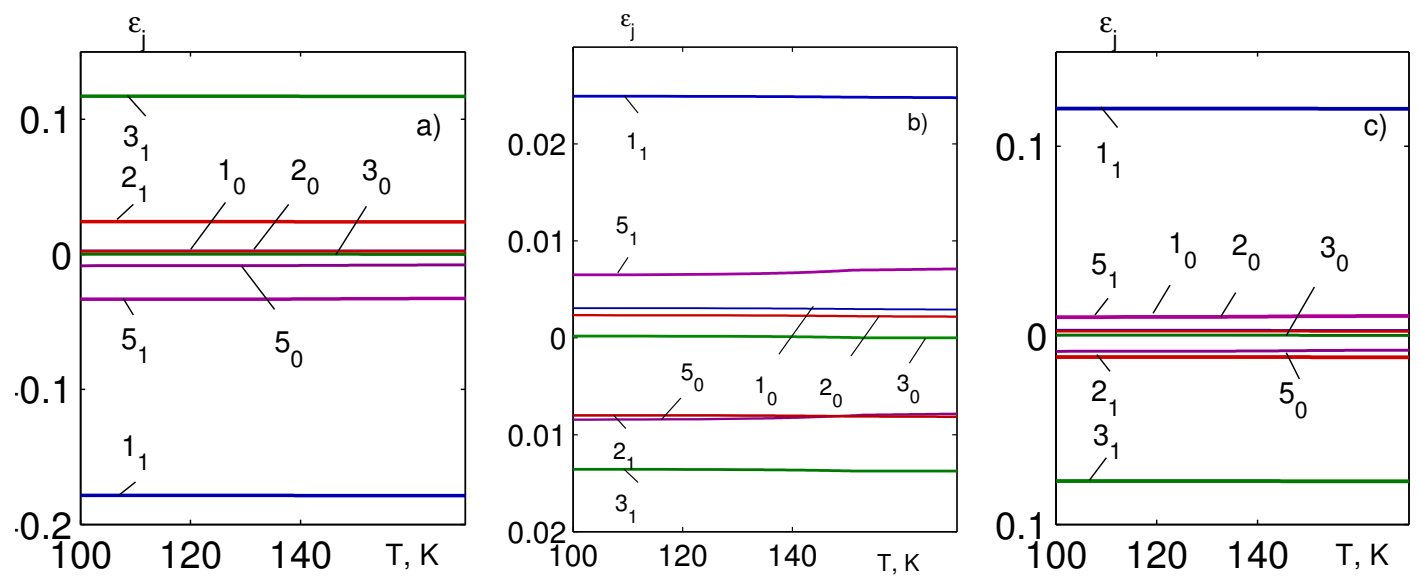

Fig. 2. The temperature dependences of the strains $\varepsilon_{1}-1, \varepsilon_{2}-2, \varepsilon_{3}-3, \varepsilon_{5}-5$ of CDP at different uniaxial pressures: $p_{1}$ $\left.\left.-\mathrm{a}), p_{2}-\mathrm{b}\right), p_{3}-\mathrm{c}\right)$. The lower index denotes the pressure values in kbar

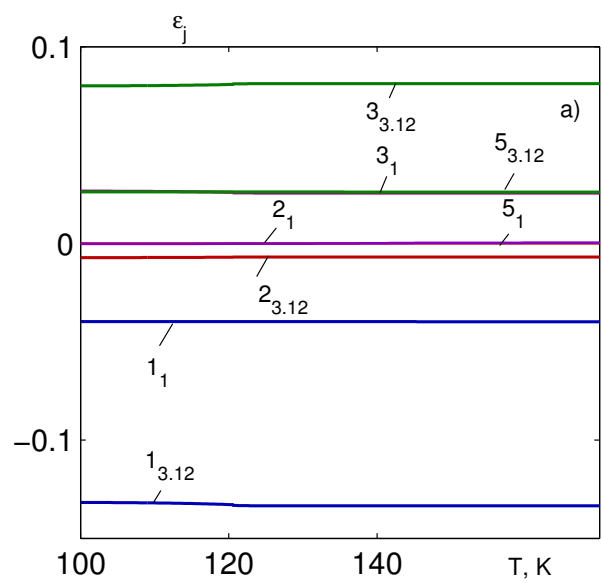

Fig. 3. The temperature dependences of the strains $\varepsilon_{1}-1, \varepsilon_{2}-2, \varepsilon_{3}-3, \varepsilon_{5}-5$ of CDP at different values of hydrostatic pressure $p_{\mathrm{h}}$. The lower index denotes the pressure values in kbar

The pressure $p_{1}$ increases the negative value of the strain $\varepsilon_{1}$ and the positive value of $\varepsilon_{3}$. The strains $\varepsilon_{2}$, $\varepsilon_{5}$ are not changed perceptibly. The uniaxial pressure $p_{2}$ increases the negative values of the strains $\varepsilon_{3}$ and $\varepsilon_{2}$, as well as the positive values of the strains $\varepsilon_{1}$ and $\varepsilon_{5}$. The pressure $p_{3}$ increases the negative values of $\varepsilon_{3}, \varepsilon_{5}$ and the positive values of $\varepsilon_{1}$ and $\varepsilon_{5}$. The magnitude of the strain $\varepsilon_{2}$ is barely changed. The pressure $p_{\mathrm{h}}$ increases the absolute values of the strains $\varepsilon_{1}$ and $\varepsilon_{3}$ and slightly increases the strain $\varepsilon_{5}$; the strain $\varepsilon_{2}$ is positive and hardly 
changes with an increase in $p_{\mathrm{h}}$. The lattice strains $\varepsilon_{j}$ depend on pressure almost linearly, in accordance with Hooke's law.

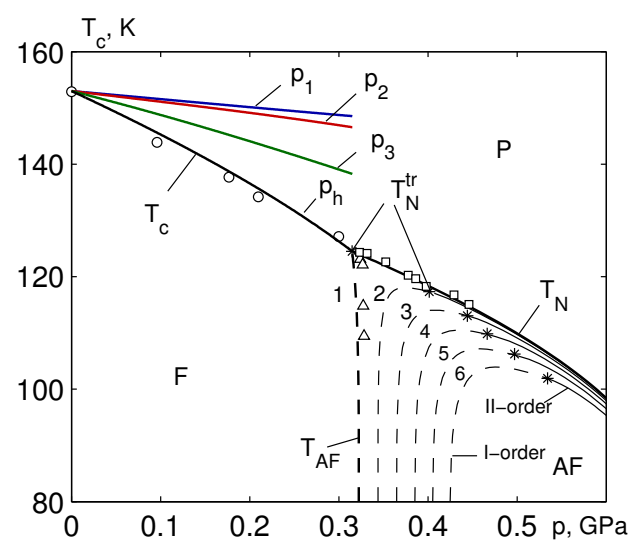

Fig. 4. The dependences of the transition temperatures of CDP on the uniaxial $p_{1} ; p_{2} ; p_{3}$ and hydrostatic pressures: between the paraelectric and ferroelectric phases $T_{c}$, between the paraelectric and antiferroelectric phases $T_{N}$, between the ferroelectric and antiferroelectric phaes $T_{\mathrm{AF}}$ at different values of the electric field $E_{2}(\mathrm{MV} / \mathrm{m}): 0.0-1,0.1-2,0.2$ $-3,0.3-4,0.4-5,0.5-6$. Symbols are the experimental data of [5]. The tricritical points $T_{N}^{\mathrm{tr}}$ (denoted by ${ }^{*}$ ) separate the curves of the first (dashed lines) and the second (solid lines) order phase transition

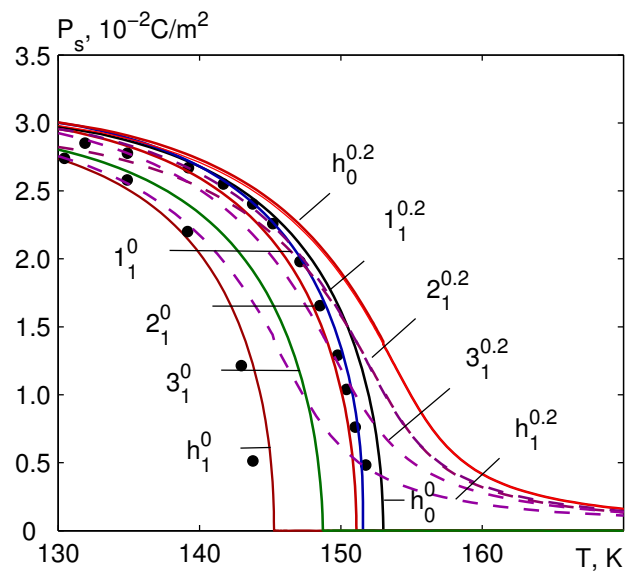

Fig. 5. The temperature dependence of the spontaneous polarization $P_{s}$ of the CDP crystal at different values of uniaxial pressures $\left(p_{1}-1 ; p_{2}-2 ; p_{3}-3\right)$, hydrostatic pressure $\left(p_{\mathrm{h}}\right)$, and electric field $E_{2}$. The upper index denotes the field value in $\mathrm{MV} / \mathrm{m}$, the lower index denotes the pressure in the units of $0.1 \mathrm{GPa}$. Dots $(\bullet)$ are the experimental data of [5]

External pressures weaken the interactions between the pseudospins. The interaction parameters $w, \nu_{1}, \nu_{2}$ decrease linearly with an increase in pressures. As a result, the transition temperature $T_{c}$ decreases (Fig. 4). From Fig. 4 it is seen that at low pressures the change in the Curie temperature under hydrostatic pressure is approximately equal to the sum of changes in the Curie temperature under uniaxial pressures.

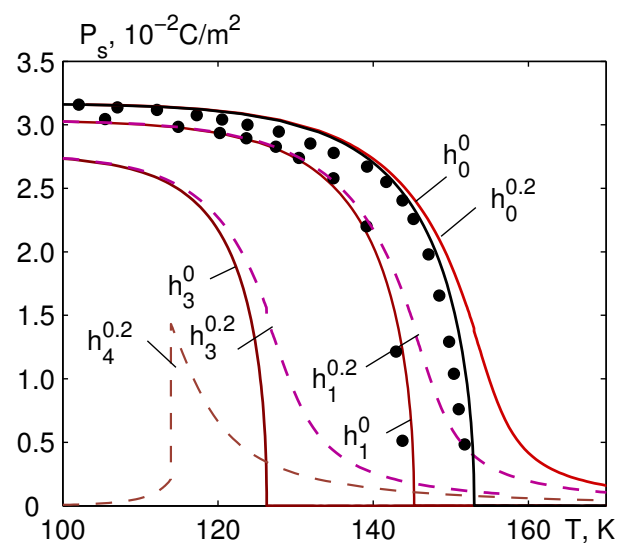

Fig. 6. The temperature dependence of the spontaneous polarization $P_{s}$ of the CDP crystal at different values of hydrostatic pressure $\left(p_{\mathrm{h}}\right)$ and electric field $E_{2}$. The upper index denotes the field value in $\mathrm{MV} / \mathrm{m}$, the lower index denotes the pressure in the units of $0.1 \mathrm{GPa}$. Dots $(\bullet)$ are the experimental data of [5]

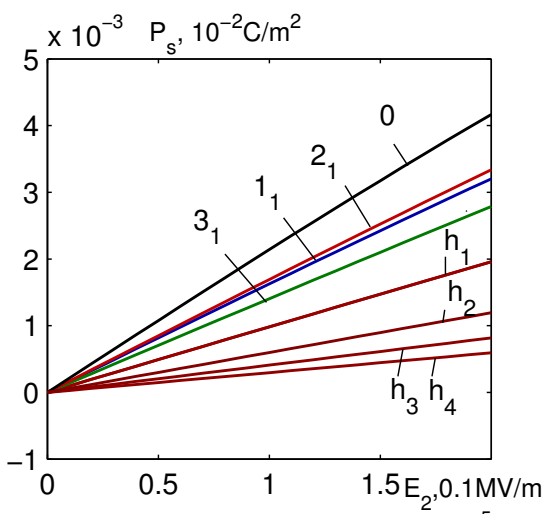

Fig. 7. Dependences of the spontaneous polarization of CDP on the electric field $E_{2}$ at different values of uniaxial pressures $\left(p_{1}-1_{1} ; p_{2}-2 p_{1} ; p_{3}-3_{1}\right)$ and hydrostatic pressure $\left(h_{1}, h_{2}, h_{3}, h_{4}\right)$. The lower index denotes the pressure in the units of $0.1 \mathrm{GPa}$

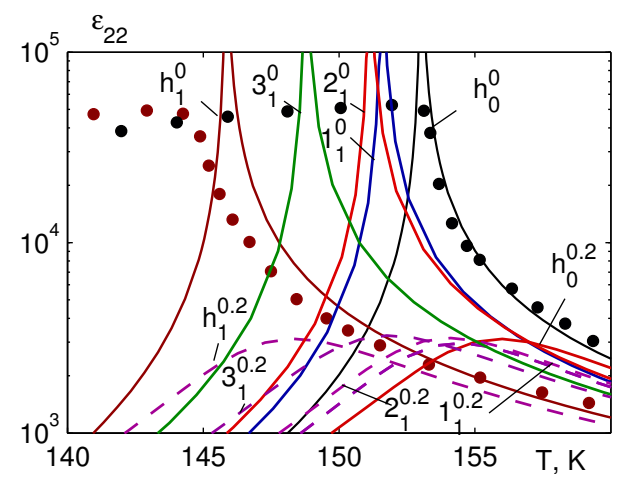

Fig. 8. The temperature dependence of the longitudinal dielectric permittivity $\varepsilon_{22}$ of CDP at different values of uniaxial pressures $\left(p_{1}-1 ; p_{2}-2 ; p_{3}-3\right)$, hydrostatic pressure $\left(p_{\mathrm{h}}\right)$, and electric field $E_{2}$. The upper index denotes the field value in $\mathrm{MV} / \mathrm{m}$, the lower index denotes the pressure in the units of $0.1 \mathrm{GPa}$. Dots $(\bullet)$ are the experimental data of [5] 
Since the transition temperature $T_{c}$ is lowered down by the pressures, the temperature curves of the spontaneous polarization $P_{s}$ (Figs. 5, 6), longitudinal dielectric permittivity $\varepsilon_{22}$ (Figs. 8, 9), piezomoduli $e_{21}, h_{21}$ (Figs. 1114), elastic constant $c_{11}$ (Figs. 16, 17), and pseudospin contribution to the heat capacity $\Delta C$ (Figs. 18, 19) all shift to lower temperatures.

In Fig. 5, we plot the temperature dependences of the spontaneous polarization $P_{s}$ of $\mathrm{CDP}$ at different values of the uniaxial pressures $\left(p_{1} ; p_{2} ; p_{3}\right)$, hydrostatic pressure $p_{\mathrm{h}}$, and electric field $E_{2}$. The temperature dependence of the spontaneous polarization $P_{s}$ at different values of hydrostatic pressure $\left(p_{\mathrm{h}}\right)$ and electric field $E_{2}$ is shown in Fig. 6, whereas the dependences of $P_{s}$ on the electric field $E_{2}$ at different values of uniaxial and hydrostatic pressures are shown in Fig. 7.

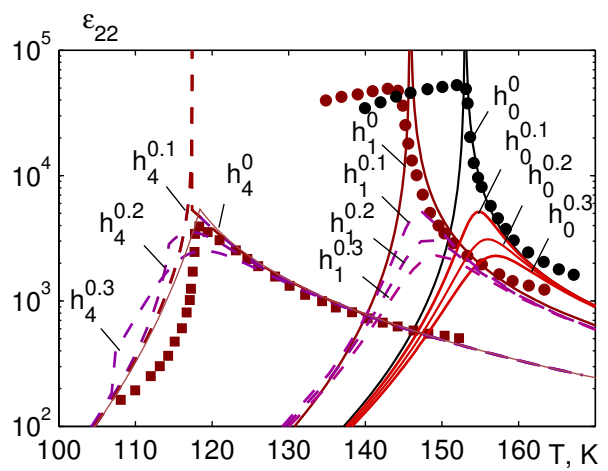

Fig. 9. The temperature dependence of the longitudinal dielectric permittivity $\varepsilon_{22}$ of CDP at different values of hydrostatic pressure $\left(p_{\mathrm{h}}\right)$ and electric field $E_{2}$. The upper index denotes the field value in $\mathrm{MV} / \mathrm{m}$, the lower index denotes the pressure in the units of $0.1 \mathrm{GPa}$. Dots $(\bullet)$ are the experimental data of [5]
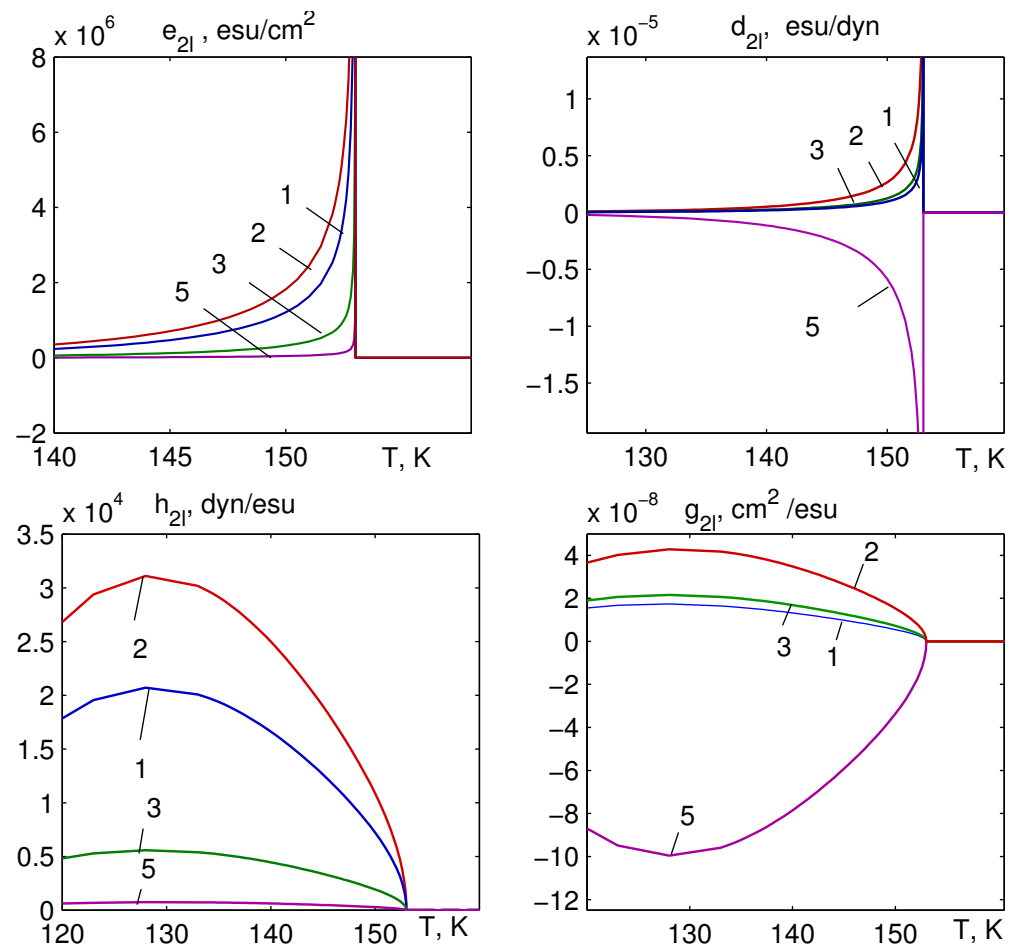

Fig. 10. The temperature dependences of the coefficients of piezoelectric stress $e_{21}-1, e_{22}-2, e_{23}-3, e_{25}-4$, coefficients of piezoelectric strain $d_{21}-1, d_{22}-2, d_{23}-3, d_{25}-4$, constants of piezoelectric stress $h_{21}-1, h_{22}-2, h_{23}-3, h_{25}-4$, and constants of piezoelectric strain $g_{21}-1, g_{22}-2, g_{23}-3, g_{25}-4$ of the CDP crystal

At low pressures, the spontaneous polarization monotonically and continuously decreases with an increase in temperature and vanishes at $T_{c}$. The phase transition at $T_{c}$ is still of the second order.

The application of the external field $E_{2}$ smears out the polarization curves, and the phase transition in CDP disappears as such.
The electric field $E_{2}$ decreases the polarization. The largest decrease is observed when the electric field and hydrostatic pressure are applied simultaneously.

The longitudinal dielectric permittivity $\varepsilon_{22}$ diverges at $T_{c}$ (figs. 8,9 ). In the presence of the electric field $E_{2}$, the permittivity $\varepsilon_{22}$ has a jump at $T_{N}$ instead of a cusp (Fig. 9). 


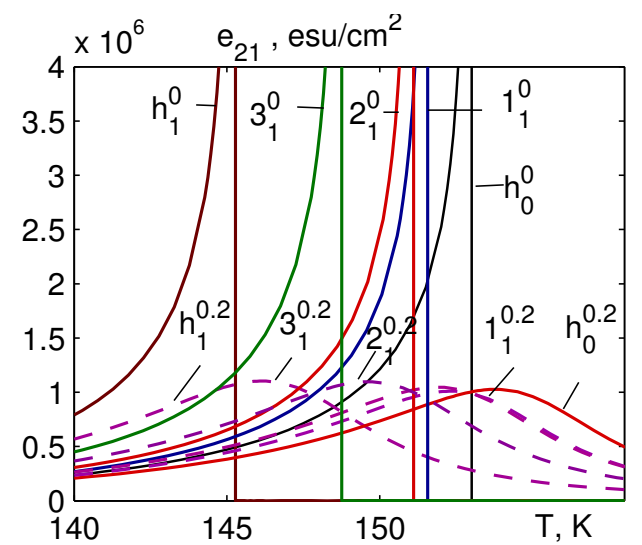

Fig. 11. The temperature dependences of the coefficient of piezoelectric stress $e_{21}$ at different values of uniaxial pressures $\left(p_{1}-1 ; p_{2}-2 ; p_{3}-3\right)$, hydrostatic pressure $\left(p_{\mathrm{h}}\right)$, and electric field $E_{2}$. The upper index denotes the field value in $\mathrm{MV} / \mathrm{m}$, the lower index denotes the pressure in the units of $0.1 \mathrm{GPa}$

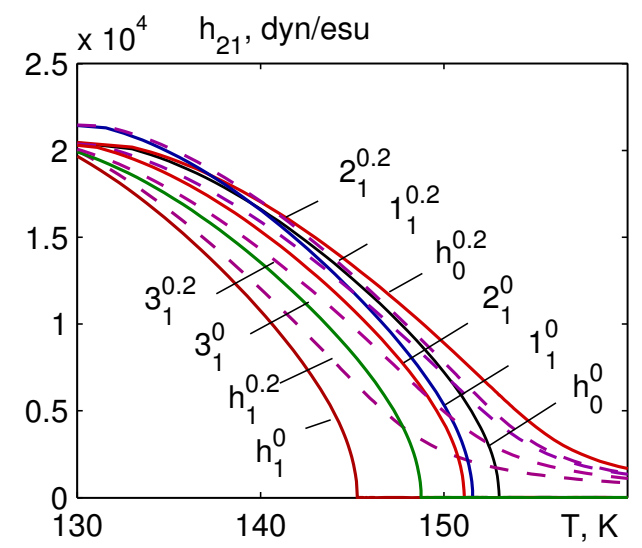

Fig. 12. The temperature dependences of the constant of piezoelectric stress $h_{21}$ at different values of uniaxial pressures $\left(p_{1}-1 ; p_{2}-2 ; p_{3}-3\right)$, hydrostatic pressure $\left(p_{\mathrm{h}}\right)$, and electric field $E_{2}$. The upper index denotes the field value in $\mathrm{MV} / \mathrm{m}$, the lower index denotes the pressure in the units of $0.1 \mathrm{GPa}$

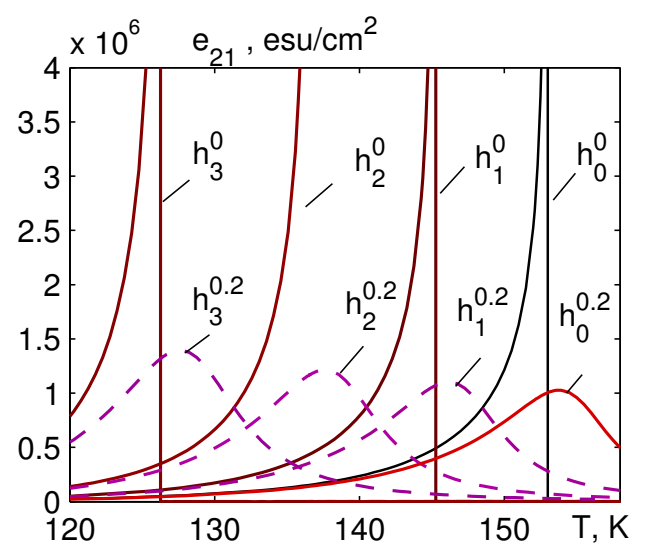

Fig. 13. The temperature dependences of the coefficient of piezoelectric stress $e_{21}$ at different values of hydrostatic pressure $\left(p_{\mathrm{h}}\right)$ and electric field $E_{2}$. The upper index denotes the field value in $\mathrm{MV} / \mathrm{m}$, the lower index denotes the pressure in the units of $0.1 \mathrm{GPa}$
In the presence of the electric field $E_{2}$ the permittivity $\varepsilon_{22}$ remains finite and has maxima, whose magnitudes decrease with an increase in the field, and which positions shift to higher temperatures.

The theoretical results agree quantitatively well with the experimental data of [5] for the case of hydrostatic pressure $p_{\mathrm{h}}$. It should be mentioned that the theory is developed for a single-domain crystal and does not take into account the reorientation of domain walls, which gives an essential contribution to the experimentally measured permittivity. That is why the calculated permittivity $\varepsilon_{22}$ in the ferroelectric phase does not agree with the experimental data.

The piezoelectric coefficients $e_{2 l}$ and $d_{2 l}$ increase with temperature and diverge at $T_{c}$ (Fig. 10), whereas the constants $h_{2 l}$ and $g_{2 l}$ have rounded maxima and approach zero at $T_{c}$.

In Figs. 11, 12 the temperature curves of the piezoelectric stress coefficient $e_{21}$ and constant $h_{21}$ of the CDP crystal are shown at different values of uniaxial pressures $\left(p_{1} ; p_{2} ; p_{3}\right)$, hydrostatic pressure $p_{\mathrm{h}}$, and electric field $E_{2}$. Their temperature curves at different values of hydrostatic pressure $\left(p_{\mathrm{h}}\right)$ and electric field $E_{2}$ are presented in Figs. 13, 14.

Hydrostatic pressure $p_{\mathrm{h}}$ increases the maximum value of the piezoelectric stress constant $h_{21}$; the influence of the electric field $E_{2}$ at lower temperatures is weak. The field $E_{2}$ smears out the curves of the piezoelectric stress constant $h_{21}$.

The field $E_{2}$ applied to the CDP crystal decreases the maximum values of the coefficient of piezoelectric stress $e_{21}$ and shifts them to higher temperatures.

The elastic constants $c_{i j}$ of CDP are virtually temperature independent, except for small jumps at $T_{c}$ (Fig. 15).

In Fig. 16, we depict the temperature dependences of the elastic constant $c_{11}$ of CDP at different values of the uniaxial pressures $\left(p_{1} ; p_{2} ; p_{3}\right)$, hydrostatic pressure $p_{\mathrm{h}}$, and electric field $E_{2}$. Its temperature dependences at different values of hydrostatic presssures $\left(p_{\mathrm{h}}\right)$ and electric field $E_{2}$ are presented in Fig. 17.

In the presence of the pressures, the jump of the elastic constant $c_{11}$ deepens. The effect of the uniaxial pressure $p_{3}$ is the strongest. Hydrostatic pressure $p_{\mathrm{h}}$ lowers down $c_{11}$. The electric field $E_{2}$ smears out the curves of the elastic constant $c_{11}$; its minimum values increase with an increase in pressures. Outside the vicinity of the transition temperature, the field $E_{2}$ does not affect the elastic constants $c_{i j}^{E}$.

The temperature curves of the pseudospin contribution to the heat capacity $\Delta C_{p}$ of CDP in presence of the uniaxial pressures $p_{i}$ exhibit jumps at the phase transition temperature and shift to lower temperatures (Fig.18). The magnitude of the jumps is slightly decreased by the pressures $p_{i}$.

The temperatures dependences of $\Delta C_{p}$ remain qualitatively unchanged by hydrostatic pressure (Fig. 19). Positions of the heat capacity maximum shift to lower temperatures, whereas the magnitude of $\Delta C_{p_{\mathrm{h}}}$ decrease, with an increase in hydrostatic pressure. 


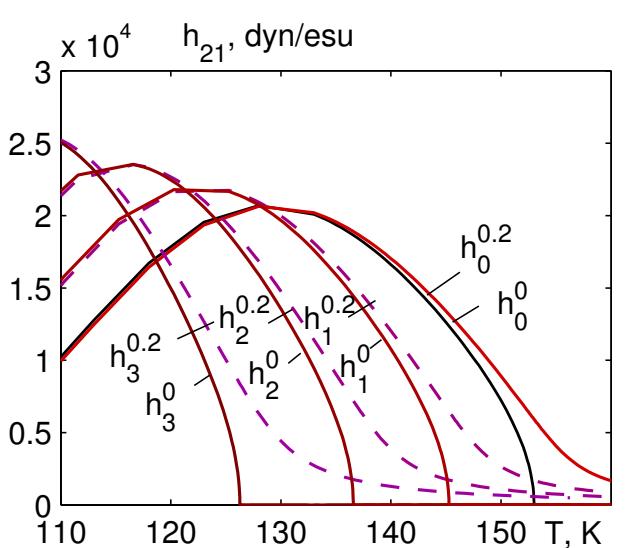

Fig. 14. The temperature dependences of the constant of piezoelectric stress $h_{21}$ at different values of hydrostatic pressure $\left(p_{\mathrm{h}}\right)$ and electric field $E_{2}$. The upper index denotes the field value in $\mathrm{MV} / \mathrm{m}$, the lower index denotes the pressure in the units of $0.1 \mathrm{GPa}$

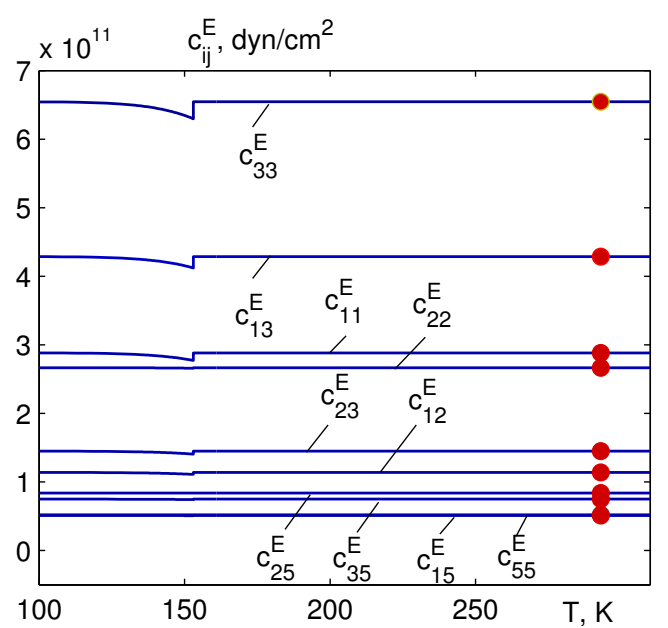

Fig. 15. The temperature dependences of the elastic constants $c_{i j}$ of CDP. Dots $(\bullet)$ are the experimental data of [25]

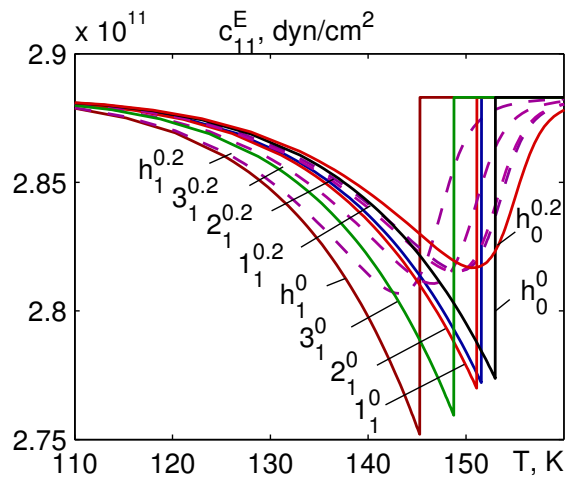

Fig. 16. The temperature dependences of the elastic constant $c_{11}$ at different values of uniaxial pressures $\left(p_{1}-1 ; p_{2}-2\right.$; $\left.p_{3}-3\right)$, hydrostatic pressure $\left(p_{\mathrm{h}}\right)$, and electric field $E_{2}$. The upper index denotes the field value in $\mathrm{MV} / \mathrm{m}$, the lower index denotes the pressure in the units of $0.1 \mathrm{GPa}$

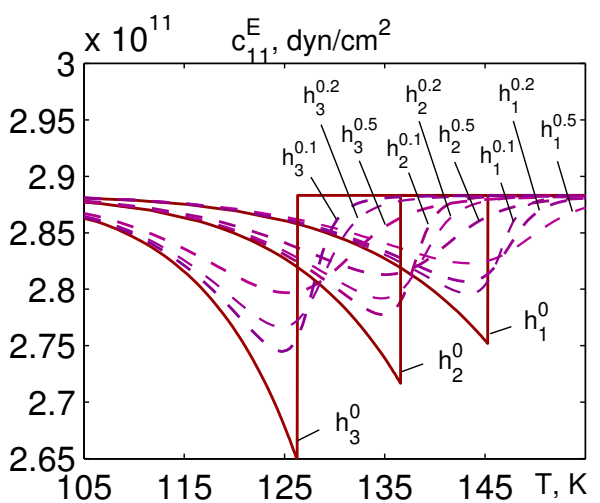

Fig. 17. The temperature dependences of the elastic constant $c_{11}$ at different values of hydrostatic pressure $\left(p_{\mathrm{h}}\right)$ and electric field $E_{2}$. The upper index denotes the field value in $M V / m$, the lower index denotes the pressure in the units of $0.1 \mathrm{GPa}$

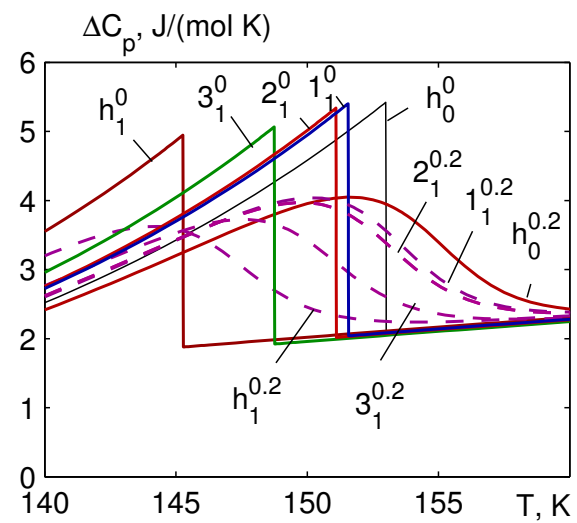

Fig. 18. The temperature dependences of the proton contribution to molar heat capacity at different values of uniaxial pressures $\left(p_{1}-1 ; p_{2}-2 ; p_{3}-3\right)$, hydrostatic pressure $\left(p_{\mathrm{h}}\right)$, and electric field $E_{2}$. The upper index denotes the field value in $\mathrm{MV} / \mathrm{m}$, the lower index denotes the pressure in the units of $0.1 \mathrm{GPa}$

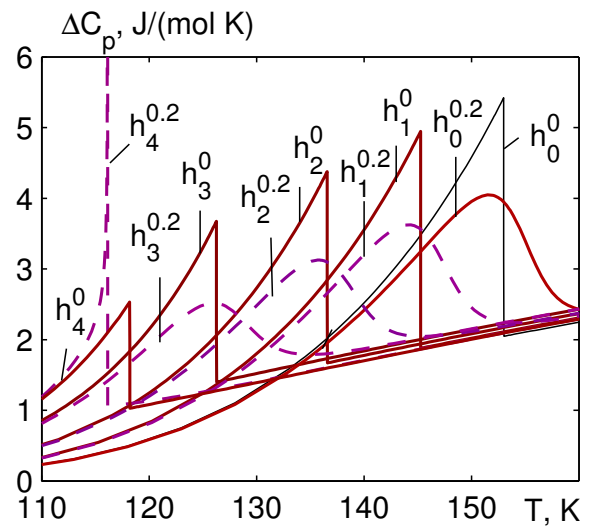

Fig. 19. The temperature dependences of the proton contribution to molar heat capacity at different values of hydrostatic pressure $\left(p_{\mathrm{h}}\right)$ and electric field $E_{2}$. The upper index denotes the field value in $\mathrm{MV} / \mathrm{m}$, the lower index denotes the pressure in the units of $0.1 \mathrm{GPa}$ 
In Figs. 18, 19, we plot the temperature dependences of the pseudospin contributions to the heat capacity $\Delta C$ of CDP in the presence of the electric field $E_{2}$, as well as of the pressures and the field $E_{2}$ simultaneously. The jump of $\Delta C$ is smeared out by the field $E_{2}$, and its maximum value is decreased. In the antiferroelectric phase, the molar heat capacity is also reduced by the field.

\section{CONCLUSIONS}

In the present work, within the framework of the modified proton ordering model for the quasi-one-dimensional $\mathrm{CsH}_{2} \mathrm{PO}_{4}$ ferroelectric with hydrogen bonds, which takes into account the contributions to the energy of the proton subsystem that are linear in the lattice strains $\varepsilon_{1}, \varepsilon_{2}$, $\varepsilon_{3}$, and $\varepsilon_{5}$, within the two-particle cluster approximation, we studied the influence of hydrostatic and uniaxi- al pressures and the longitudinal electric field $E_{2}$ on the phase transition and physical characteristics of this ferroelectric. It has been established that the uniaxial and hydrostatic pressure change the lattice strains $\varepsilon_{i}, \varepsilon_{5}$, thereby changing the transition temperature and other characteristics of the crystal.

Since the transition temperatures $T_{c}$ are decreased by the pressures, the temperature curves of the spontaneous polarization $P_{s}$, longitudinal dielectric permittivity $\varepsilon_{22}$, piezomoduli $e_{21}, d_{21}, h_{21}, g_{21}$, elastic constant $c_{11}$, and pseudospin contribution to the heat capacity $\Delta C$ shift to lower temperatues under pressures.

At pressures $p<p_{c}$ the external field smears out the phase transition. At $p>p_{c}$ the field lowers down the transition temperature $T_{N}$ and discontinuously increases the permittivity $\varepsilon_{22}$, elastic constant $c_{11}$, and pseudospin contribution to the heat capacity $\Delta C$ in the antiferroelectric phase at $E_{2}=0.1 \mathrm{MV} / \mathrm{m}$.
[1] H. Matsunaga, K. Itoh, E. Nakamura, J. Phys. Soc. Jpn. 48, 2011 (1980); https://doi.org/10.1143/JPSJ. 48. 2011.

[2] K. Itoh, T. Hagiwara, E. Nakamura, J. Phys. Soc. Jpn. 52, 2626 (1983); https://doi.org/10.1143/JPSJ.52. 2626.

[3] Y. Iwata, N. Koyano, I. Shibuya, J. Phys. Soc. Jpn. 49, 304 (1980); https://doi.org/10.1143/JPSJ .49.304.

[4] Y. Iwata et al, J. Phys. Soc. Jpn. 63, 4044 (1994); https : //doi.org/10.1143/JPSJ.63.4044.

[5] N. Yasuda et al, Phys. Rev. Lett. 41, 1311 (1978); https : //doi.org/10.1103/PhysRevLett.41.1311.

[6] N. Yasuda, S. Fujimoto, M. Okamoto, H. Shimizu, K. Yoshino, Y. Phys. Rev. B 20, 2755 (1979); https: //doi.org/10.1103/PhysRevB.20.2755.

[7] P. J. Schuele, R. A. Thomas. Jap. J. Appl. Phys. 24, 935 (1985); https://doi.org/10.7567/JJAPS. 24S2.935.

[8] P. J. Schuele, V. H. Schmidt, Phys. Rev. B 39, 2549, (1989); https://doi.org/10.1103/PhysRevB.39. 2549.

[9] Yu. Kobayashi et al, Ferroelectrics 285, 83 (2003); http s://doi.org/10.1080/00150190390205924.

[10] K. Gesi, K. Ozawa. Jpn. J. Appl. Phys. 17, 435 (1978); https://doi.org/10.1143/JJAP. 17.435.

[11] N. B. Brandt, S. G. Zhukov, V. A. Kulbachinskii, P. S. Smirnov, B. A. Strukov, Fiz. Tverd. Tela 28, 3159 (1986).

[12] E. Magome, S. Tomiaka, Y. Tao, M. Komukae. J. Phys. Soc. Jpn. 79, 025002 (2010); https://doi.org/10.114 3/JPS J.79.025002.

[13] Ya. Shchur, Phys. Rev. B 74, 054301 (2006); https:// doi.org/10.1103/PhysRevB.74.054301.

[14] Ya. Shchur, Phys. Status Solidi B 244, 569 (2007); http s://doi.org/10.1002/pssb. 200642176.

[15] J. Lasave, P. Abufager, S. Koval, Phys. Rev. B 93,
134112 (2016); https://doi.org/10.1103/PhysRevB.9 3.134112.

[16] Ya. Shchur, T. Bryk, I. Klevets, A.V. Kityk, Comput. Mater. Sci. 111. 301 (2016); https://doi.org/10.101 6/j.commatsci.2015.09.014.

[17] B. Van Troeye et al, Phys. Rev. B 95, 024112 (2017); https://doi.org/10.1103/PhysRevB.95.024112.

[18] R. Blinc, F. C. SaBaretto, J. Chem. Phys. 72, 6031 (1988); https://doi.org/10.1063/1.439058.

[19] I. V. Stasyuk et al., preprint ICMP-91-4R (Lviv, 1991).

[20] K. Deguchi, E. Okaue, S. Ushio, E. Nakamura, K. Abe, J. Phys. Soc. Jpn. 53, 3074 (1984); https://doi.org/ 10.1143/JPS J.53.3074.

[21] R. R. Levitskii, I. R. Zachek, A. S. Vdovych, Phys. Chem. Solid State 13, 40 (2012).

[22] R. R. Levitskii, I. R. Zachek, A. S. Vdovych, J. Phys. Stud. 16, 4702 (2012); https://doi.org/10.30970/jps .16 .4702 .

[23] A. S. Vdovych, I. R. Zachek, R. R. Levitskii, Condens. Matter Phys. 23, 33702 (2020); https://doi .org/10.5 488/CMP. 23.33702.

[24] K. Imai, J. Phys. Soc. Jpn. 52, 3960 (1983); https:// doi .org/10.1143/JPS J. 52.3960.

[25] S. Praver, T. F. Smith, T. R. Finlayson, Aust. J. Phys. 3863 (1985); https://doi.org/10.1071/PH850063.

[26] Y. Uesu, J. Kobayashi, Phys. Status Solidi A 34, 475 (1976); https://doi.org/10.1002/pssa. 2210340207.

[27] R. J. Nelmess, R. N. P. Choudhary, Solid State Commun. 26, 823 (1978); https://doi.org/10.1016/0038-109 $8(78) 90751-2$

[28] B. C. Fraser, D. Semmingsen, W. D. Ellenson, D. Shirane. Phys. Rev. B 20, 2745 (1979); https://doi.org/ 10.1103/PhysRevB.20.2745. 


\title{
ВПЛИВ ОДНОВІСНИХ ТА ГІДРОСТАТИЧНОГО ТИСКІВ І ПОЗДОВЖНЬОГО ЕЛЕКТРИЧНОГО ПОЛЯ НА ФАЗОВІ ПЕРЕХОДИ ТА ТЕРМОДИНАМІЧНІ ХАРАКТЕРИСТИКИ КВАЗІОДНОВИМІРНОГО СЕГНЕТОЕЛЕКТРИКА $\mathrm{CsH}_{2} \mathrm{PO}_{4}$
}

\author{
А. С. Вдович ${ }^{1}$, Р. Р. Левицький ${ }^{1}$, І. Р. Зачек ${ }^{2}$, А. П. Моїна ${ }^{1}$ \\ ${ }^{1}$ Інститут фізики конденсованих систем НАН Украӥни, \\ вул. Свениіцького, 1, Лъвів, 79011, Україна, \\ ${ }^{2}$ Націоналъний університет "Лъвівсъка політехніка", \\ вул. С. Бандери 12, 79013, Лъвів, Украӥна
}

Квазіодновимірний кристал із водневими зв'язками $\mathrm{CsH}_{2} \mathrm{PO}_{4} \in$ сегнетоелектриком типу ладбезлад. Фазовий перехід із високотемпературної парафази в низькотемпературну сегнетофазу пов'язаний з упорядкуванням протонів на водневих зв'язках.

Запропоновано модифіковану двопідграткову псевдоспінову модель сегнетоелектрика $\mathrm{CsH}_{2} \mathrm{PO}_{4}$, у якій положенням протонів приписано ефективні дипольні моменти та псевдоспіни, а кристал розглядається як система взаємодійних псевдоспінів. Ця модель ураховує лінійні за деформаціями гратки $\varepsilon_{1}, \varepsilon_{2}, \varepsilon_{3}$ i $\varepsilon_{5}$ внески в енергію протонної підсистеми, а також залежність ефективних дипольних моментів псевдоспінів від параметрів упорядкування, що дозволяє узгодити ефективні дипольні моменти в сегнето- і парафазі.

У межах цієї моделі в наближенні двочастинкового кластера за короткосяжними й середнього поля за далекосяжними взаємодіями досліджено поведінку спонтанної поляризації, поздовжньої діелектричної проникності, п'єзоелектричних, пружних характеристик і молярної теплоємності. Вивчено вплив гідростатичного та одновісних тисків, а також поздовжнього електричного поля на фазовий перехід та фізичні характеристики кристала. Отримано задовільний кількісний опис відповідних експериментальних даних.

Показано, що під дією одновісних тисків, а також гідростатичного тиску, меншого за критичний $p<p_{c}$, понижується температура фазового переходу пара-сегнетофаза. При цьому температурні залежності різних термодинамічних характеристик якісно подібні, як за відсутності тисків. Зовнішнє електричне поле за тисків $p<p_{c}$ розмиває фазовий перехід.

За гідростатичного тиску $p>p_{c}$ виникає перехід в антисегнетофазу. Електричне поле за $p>$ $p_{c}$ понижує температуру фазового переходу пара-антисегнетофаза й збільшує проникність $\varepsilon_{22}$ в антисегнетофазі. Достатньо сильне поле може змінити рід фазового переходу в точці $T_{N}$ iз другого на перший.

Найсильніший вплив поля на розраховані характеристики $є$ поблизу критичного гідростатичного тиску $p_{c}$; із пониженням температури кристал $\mathrm{CsH}_{2} \mathrm{PO}_{4}$ переходить спершу з парафази в антисегнетофазу, а за подальшого пониження температури - 3 антисегнетофази в сегнетофазу. Поздовжнє поле $E_{2}$ збільшує критичний гідростатичний тиск $p_{c}$ у кристалі $\mathrm{CsH}_{2} \mathrm{PO}_{4}$.

Ключові слова: сегнетоелектрики, діелектрична проникність, п'єзоелектричні коефіцієнти, вплив тиску, вплив електричного поля. 